\title{
Relation between volume and outcome for patients with severe sepsis in United Kingdom: retrospective cohort study
}

\author{
(®) $\Theta$ OPEN ACCESS
}

\author{
Jason Shahin assistant professor ${ }^{1}$ clinical research associate ${ }^{2}$, David A Harrison senior statistician ${ }^{2}$, \\ Kathryn M Rowan director ${ }^{2}$
}

${ }^{1}$ McGill University, Department of Critical Care, Department of Medicine, Respiratory Division, 1650 Cedar Avenue, Montreal, Quebec, Canada H3G 1A4; Intensive Care National Audit \& Research Centre (ICNARC), London WC1H 9HR, UK

\begin{abstract}
Objective To evaluate whether a relation exists between volume and outcome for admissions with severe sepsis to adult general critical care units in the United Kingdom.

Design Retrospective cohort study using data from a pooled case mix and outcome database.

Setting Adult general critical care units participating in the case mix programme.

Participants Consecutive admissions to participating units for the years 2008-09 meeting objective, standardised criteria for severe sepsis.

Main outcome measures Mortality at ultimate discharge from acute hospital.

Results The primary exposure was volume of admissions with severe sepsis per unit per year. A multivariable logistic regression analysis, using generalised estimating equations, was used to assess the association between volume, modelled using fractional polynomials, and ultimate acute hospital mortality while adjusting for potential confounders. No relation was seen between volume and outcome for admissions with severe sepsis to adult, general critical care units in the UK. Subgroup analyses tested for interactions between the effect of volume and acute severity of illness or receipt of mechanical ventilation. No significant interactions were found.
\end{abstract}

Conclusions This study showed no relation between volume and outcome in admissions with severe sepsis treated in adult general critical care units in the UK.

\section{Introduction}

Over the past three decades, studies have evaluated the relation between the volume of cases treated and patients' outcomes for a variety of surgical and medical conditions. ${ }^{1}$ A strong association was found for complex surgical procedures, such as repair of abdominal aortic aneurysms and surgery for specific cancers and paediatric cardiac conditions. For medical conditions such as AIDS and myocardial infarction, treatment in high volume centres has also been associated with improved outcome. ${ }^{1}$ Several studies have evaluated the volume-outcome relation for critically ill patients. ${ }^{2}$ These studies have included all critically ill patients, as well as selected groups of patients, such as those who were mechanically ventilated and those with severe sepsis. Two alternative hypotheses have been put forward to explain the underlying mechanism for volume-outcome relations: "practice makes perfect" and "selective referral." ${ }^{, 3}$ In the case of acutely ill patients in critical care units, the most likely mechanism would be "practice makes perfect," as little scope exists for critically ill patients to choose where they are treated.

Admissions with severe sepsis make up roughly one quarter of all admissions to adult general critical care units in the United Kingdom. Over the past decade, the incidence of severe sepsis in the UK increased from 46 per 100000 to 60 per 100000 in 2009 (personal correspondence from the Intensive Care National Audit \& Research Centre). ${ }^{4}$ As the incidence of admissions with severe sepsis increases, the costs of caring for these critically ill patients, in terms of infrastructure, personnel, and therapeutics, will put an enormous strain on the healthcare system. With this and other increased demand for critical care beds, alternative models for organising critical care services are being considered. One such model calls for centralisation of care, whereby critically ill patients at higher risk are routinely transferred from smaller to larger designated centres for more specialised care. ${ }^{2}$ Centralisation has been successfully carried out in the UK for paediatric critical care and, more recently, for trauma ${ }^{5-9}$ but it does not exist for adult general critical care. The rationale behind centralisation is twofold. Firstly, by focusing 
allocated healthcare funds on only a smaller number of specialised centres, the financial burden can be contained; secondly, health outcomes are potentially improved by treating patients in high volume facilities. ${ }^{6} 10$

Two previous studies have assessed the volume-outcome relation for admissions with severe sepsis to adult general critical care and showed a lower hospital mortality for admissions treated in units with higher annual volume. ${ }^{11}{ }^{12}$ These studies, however, were done outside the UK and included relatively few critical care units and admissions. Therefore, the existence of a relation between volume and outcome for admissions with severe sepsis to adult general critical care units in the UK remains unclear. In view of the need for the NHS to understand volume-outcome relations to inform service delivery and organisation of care, ${ }^{13}$ this study evaluated whether a volume-outcome relation exists for admissions with severe sepsis in adult general critical care units in the UK.

\section{Methods \\ Study design}

We did a secondary analysis of the case mix programme database. ${ }^{13}$ This database contains pooled case mix and outcome data on consecutive admissions to adult general (mixed medical/surgical) critical care units (that is, stand alone intensive care units and combined intensive care/high dependency units) in England, Wales, and Northern Ireland that are collected as part of the national clinical audit coordinated by the Intensive Care National Audit \& Research Centre (ICNARC). The database contains raw physiological and diagnostic data needed for the acute physiological and chronic health evaluation (APACHE) II and ICNARC risk prediction models, ${ }^{14}{ }^{15}$ together with demographic, outcome, and activity data. Trained data collectors collect case mix programme data prospectively and abstract them retrospectively. Data undergo extensive validation, both locally and centrally, before being pooled in the central database. Details of data collection and validation have been reported previously, ${ }^{16}$ and the case mix programme database has been independently assessed to be of high quality. ${ }^{17}$

\section{Patient selection}

We extracted data for 2008-09, selecting patients aged 16 years or older who met the criteria for severe sepsis in the first 24 hours after admission to a critical care unit. Using raw physiological and diagnostic data, we considered severe sepsis to be present if the admission had data indicating evidence of three or more systemic inflammatory response syndrome criteria, infection, and evidence of at least one organ dysfunction (see supplementary table A for precise definitions).

We excluded critical care units with less than six months of data in 2008-09 and admissions transferred either directly into a unit from another critical care unit or transferred out of a unit directly to another critical care unit within 24 hours. In addition, we excluded subsequent readmissions to the same critical care unit during the same acute hospital stay.

\section{Exposure, outcome, and confounding variables}

The primary exposure of interest was volume of admissions with severe sepsis per unit per year. We included all admissions with severe sepsis (that is, before above exclusions) in the calculation of volume. For critical care units contributing less than one year of data (but more than six months) in 2008-09, we extrapolated the number of admissions with severe sepsis per year from available data. For the primary analysis, we used fractional polynomials (degree 2 ) to model volume. For reporting descriptive statistics and for secondary analyses, we grouped volume both by quarters of critical care units and by quarters of admissions. The primary outcome was ultimate acute hospital mortality, defined as death before final discharge from the acute hospital, and included deaths after direct transfer to another acute hospital from the acute hospital housing the critical care unit.

For critical care admissions, data were available on age, sex, ethnicity, acute severity of illness, severe medical history, location before admission, mechanical ventilation, length of stay, and hospital discharge location. We categorised ethnicity as white or non-white. We used the ICNARC physiology score from the ICNARC model to measure acute severity of illness. ${ }^{14} 15$ We defined medical history by severe comorbidities, using the APACHE II method (severe cardiovascular disease, severe respiratory disease, renal disease, chronic liver disease, haematological malignancy, metastatic disease, immunological dysfunction), in the six months before admission ${ }^{14}$; by activities of daily living in the six months before admission; and by cardiac arrest in the 24 hours before admission. We categorised location before admission as operating theatre, hospital ward, high dependency unit, or emergency department. We further categorised admissions from the operating theatre as following emergent/urgent or elective/scheduled surgery. We defined mechanical ventilation by receipt of mechanical ventilation either at admission to or in the first 24 hours after admission to the critical care unit. We divided length of stay into critical care unit and hospital stay, where hospital stay included continuous stay in acute hospital, even if transferred. We defined hospital discharge location as discharge to home, residential place of work/education, or non-health related institution; nursing home/nursing home equivalent; short term rehabilitation; long term rehabilitation; other health related institution; or

hospice/hospice equivalent. Finally, hospital type was available, defined by the hospital's reported university affiliation. We used age, sex, acute severity of illness, severe medical history, location before admission, and hospital type to adjust for confounding.

\section{Sample size}

After adjustment for the cluster effect, at least 26500 admissions and 42 critical care units per group would be needed to detect a difference in mortality of $5 \%$ from a baseline mortality of $40 \%$ with an $\alpha$ level of 0.05 and $90 \%$ power. This sample size assumes an exposure variable with four groups and a design effect of 3.3 (intra-cluster correlation coefficient 0.014 ), which was calculated from case mix programme data from 2007.

\section{Statistical analysis}

We analysed baseline characteristics by quarters of volume. We explored all variables with frequency distributions and cross tabulation of exposure with outcome.

We used Stata version 10.1 for statistical analyses. We did a multivariable analysis to assess the effect of volume (admissions with severe sepsis per unit per year) on outcome (ultimate acute hospital mortality) while adjusting for a priori selected confounders. All of the variables were entered into the model simultaneously with no statistical selection process applied. A logistic regression model was fitted with generalised estimating equations and robust standard errors to adjust for clustering of outcome at the critical care unit level. We did hypothesis tests using Wald tests. We used multivariable fractional polynomial 
modelling (degree 2) to select the best functional form for continuous factors (volume, ICNARC physiology score, and age)..$^{18}$

We used interaction tests to explore two subgroup analyses, selected a priori. The first, between volume and acute severity of illness, tested the hypothesis that sicker admissions may have derived greater benefit from being treated in higher volume units. The second, between volume and mechanical ventilation, tested the hypothesis that mechanically ventilated admissions may have derived greater benefit from being treated in higher volume units. We used the "MPFIgen" procedure to explore interactions with continuous variables. ${ }^{18}$ Briefly, we constructed multiplicative interaction terms between the fractional polynomial transformations selected in the main model without interactions. The model was then refitted with the interaction terms added and all interaction terms jointly tested using a Wald test.

As secondary analyses, and in the absence of a gold standard for defining volume, ${ }^{3}$ we repeated the above analyses with volume groups divided into quarters both by number of critical care units and by number of admissions and with ICNARC physiology score and age also fitted as categorical variables. The secondary analyses used random effects logistic regression models.

\section{Results}

Between 1 January 2008 and 31 December 2009, the case mix programme database contained 162648 admissions to 181 adult, general critical care units in 181 acute hospitals; raw data for $33955(20.9 \%)$ admissions met the standard objective definition used for determining severe sepsis (fig $1 \Downarrow$ ). After exclusion of 11 units contributing less than six months' worth of data, 33 538 admissions from 170 units were available for calculating the volume of admissions with severe sepsis per unit per year. Following the calculation of volume, we excluded readmissions within the same hospital stay $(\mathrm{n}=995,2.9 \%)$, direct transfers into a unit from another critical care unit $(\mathrm{n}=1692,5.0 \%)$, and direct transfers out to another critical care unit within 24 hours $(\mathrm{n}=124,0.4 \%)$. The final study sample available for analysis was 30727 admissions with severe sepsis to 170 units, representing more than $80 \%$ of adult general critical care units in the UK.

The proportion of all admissions that met systemic inflammatory response syndrome, infection, and organ dysfunction criteria did not vary markedly across quarters of volume (table $1 \Downarrow$ ). The main factor driving the variation in volume of severe sepsis admissions was the number of beds in the critical care unit (table $2 \Downarrow$ ), and this was also associated with university status. Half of the admissions with severe sepsis met four systemic inflammatory response syndrome criteria, and most had more than one organ dysfunction (table $1 \Downarrow$ ).

Depending on the volume grouping, the number of units, admissions, and median volume differed (table $2 \Downarrow$ ). In grouping by quarters of units, the groups had roughly equal number of units and increasing number of admissions in the higher volume groups. In grouping by quarters of admission, the groups had roughly similar number of admissions with decreasing number of units. The median volume was consistently lower in the unit quarter groups than in the admission quarter groups.

Admissions with severe sepsis were slightly older and had a higher level of acute severity of illness compared with all admissions to units to critical care units in the case mix programme database (mean age 60 years, median ICNARC physiology score 16) (table $3 \Downarrow$ ). Most admissions had no previous severe comorbidities and, before admission, were able to carry out activities of daily living without assistance.

Admissions were predominantly from the hospital ward, and more than half were mechanically ventilated in the first 24 hours after admission to the unit. Admissions were resource intensive, with long lengths of unit and hospital stay and high unit and acute hospital mortality (table $3 \Downarrow$ ).

Admissions in the highest volume quarter had the lowest ICNARC physiology score but were more likely to have had a previous illness (table $3 \Downarrow$ ). Crude unit mortality and ultimate acute hospital mortality were lowest for admissions in the highest quarter. Grouping volume by quarters of admissions, instead of by quarters of critical care units, did not substantially change any of the baseline variables across the quarters.

The multivariable analysis showed that, after adjustment for confounders, although the volume-outcome relation was significantly non-linear $(\mathrm{P}<0.001$ for fractional polynomial degree 2 compared with linear), no significant overall relation existed between volume and acute hospital mortality $(\mathrm{P}=0.65)$ (fig $2 \Downarrow$ ). However, the $95 \%$ confidence intervals around the estimated odds ratios do not rule out a magnitude of effect that may be considered clinically important. We also found no significant interaction between volume and acute severity of illness $(\mathrm{P}=0.46)$ (fig $3 \Downarrow)$ or receipt of mechanical ventilation $(\mathrm{P}=0.42)$ (fig $4 \Downarrow$ ).

Secondary analyses grouping volume by quarters of critical care units supported the finding of no association between volume and outcome after adjustment for confounders (supplementary table B). Grouping volume by quarters of admissions, instead of critical care units, did not change the association. We also found no significant interaction between volume and acute severity of illness, when both volume and ICNARC physiology score were grouped by quarters (supplementary table $\mathrm{C}$ ). When we grouped volume by quarters of critical care units, we found a significant interaction between volume and receipt of mechanical ventilation, with higher adjusted odds of mortality in the lowest quarter of volume for the mechanically ventilated admissions (table $4 \Downarrow$ ). However, this interaction did not remain when we grouped volume by quarters of admissions. We also saw a similar magnitude of odds ratios in the highest severity of illness quarter, although we had less power to detect an interaction with severity of illness owing the smaller sample sizes per group.

\section{Discussion}

This study found no relation between volume and outcome for all admissions with severe sepsis to adult general critical care units in the UK. Subgroup analyses found no significant interaction between volume and severity of illness or receipt of mechanical ventilation.

\section{Strengths and weaknesses of study}

The study has several strengths: the completeness, coverage, and representativeness of the data analysed; the application of an objective, standardised method to determine an admission as having severe sepsis; the ability to adjust extensively for case mix; and the adjustment for clustering of patients' outcomes. Failure to adjust for clustering of outcomes in volume-outcome studies has been previously shown to lead to bias through an overestimation of the effect estimates. ${ }^{19}$

Several limitations are also worth noting. Firstly, bias may have arisen from misclassification of cases of severe sepsis. We used physiological and diagnostic data from the first 24 hours of 
admission to the critical care unit to identify admissions with severe sepsis. As a result, patients with severe sepsis whose primary or secondary reasons for admission were misclassified as non-infectious could have been missed. Additionally, admissions with diseases other than severe sepsis that fulfil the systemic inflammatory response syndrome criteria (such as acute pancreatitis) may have been misclassified. Despite the potential for bias, we based identification of severe sepsis on raw physiological and diagnostic data using objective, standardised criteria across units. Secondly, as for any observational study, the possibility of residual confounding exists. Given that the major confounder is units' case mix, and adjustment was carried out using the validated ICNARC physiology score from the ICNARC model plus other known confounders, residual confounding is unlikely to be a major source of bias. The final limitation is the use of hospital mortality instead of mortality at a specific time point as the main study outcome. Use of data on location after discharge from acute hospital, however, goes some way towards minimising the limitation associated with an event based, rather than a time based, measure (for example, 30 day mortality).

\section{Strengths and weaknesses in relation to other studies}

The results of this study are at odds with those of previous published studies. The two studies that looked at admissions with severe sepsis to critical care units both showed a reduction in hospital mortality in admissions treated in units with higher volumes. The study by Reinikainen et al was a retrospective analysis of 452 admissions with severe sepsis in 24 Finnish units. ${ }^{12}$ After adjusting for severity of illness, they found an increased risk of death associated with treatment in smaller as compared with larger units. However, the study had two important limitations: the number of admissions and units enrolled was small, and the analysis did not take into account the clustering of patients' outcomes. The exposure variable for this study was a combination of size and teaching status of hospital/unit, rather than volume. Volume varied across the three exposure groups (median volumes from the four month study period were 10,15 , and 29); however, even allowing for the short study duration, these volumes are all towards the low end of the volume distribution in our study. The second study analysed 4605 admissions with severe sepsis treated in 28 Dutch units. ${ }^{11}$ This study found a lower mortality for admissions treated in higher volume units. The authors accounted for clustering of outcomes by using generalised estimation equations. To overcome the potential bias of the small sample of units, they used a leverage analysis. Despite the leverage analysis showing that their results were not due to data from one specific unit, given the relatively small sample of units in the study, the possibility of a group of units biasing these results still exists. The volume of severe sepsis admissions in this study was lower on average than in our study but with similar variability across units (mean $73(\mathrm{SD} 44) v 119(50))$.

Our study confirms the results of two small volume-outcome studies done in the UK and based on all admissions to adult general critical care units. ${ }^{20}{ }^{21}$ After adjustment for case mix, no relation between volume and mortality was found. These studies were limited mainly by the lack of adjustment for clustering of data. Furthermore, the analysis was done on earlier, smaller samples of the same database as used for our study.

The possible finding of a threshold effect among mechanically ventilated patients admitted with severe sepsis would be in keeping with the literature on mechanically ventilated patients. Three studies, to date, have looked at the volume-outcome relation in all mechanically ventilated admissions admitted to a critical care unit. ${ }^{22-24}$ Two of these studies showed a volume-outcome relation, but the third study failed to do so. The negative study did not adequately adjust for differences in case mix, which may explain the discrepant results.

\section{Meaning of study}

Several reasons might explain the lack of a volume-outcome relation in this study. Firstly, differences in outcomes between high and low volume units might become apparent only when the difference in volumes between groups is very large. Secondly, the units' patterns of practice may be altered by the information they receive from the case mix programme. The primary role of the case mix programme is to provide participating units with comparative data on their patients' outcomes. The units can then use the information to make changes to their services and programmes to improve their care. As the case mix programme has been in place for more than a decade, many units are likely to have made improvements, narrowing any existing gap in outcomes between high and low volume units. Thirdly, the wide dissemination of treatment guidelines (six hour resuscitation and 24 hour management bundles), through the surviving sepsis campaign, may have led to greater standardisation of practice across critical care units. ${ }^{25}$

The results of the subgroup analysis suggest a possibility that patients with greater severity of illness, receiving mechanical ventilation, or both may derive benefit from treatment in a higher volume unit. The discrepancy between the results from the two different volume groupings and from the fractional polynomial modelling may be explained by several mechanisms. The significant interaction result may be a false positive result that is an artefact of the arbitrary choice of thresholds for grouping units, supporting the use of continuous modelling over a categorical approach for the primary analysis. Alternatively, this may represent a genuine threshold effect-a functional form that fractional polynomials, and other continuous non-linear models, are not ideally placed to detect. ${ }^{26}$ The analysis that grouped volume by quarters of units would have more power to detect such a threshold effect owing to the equal numbers of units in each group and the smaller median volumes in the lower quarters in the unit groupings. No gold standard exists for grouping volume, as shown by both the critical care literature and the general medical/surgical literature, which commonly use both methods. ${ }^{3}$

The results of this study have important implications for the organisation of critical care services. People have suggested that the treatment of critically ill patients should be centralised, with care carried out in specifically designated centres. The evidence supporting centralisation of critical care comes from successful analogous systems in trauma, paediatric, and neonatal care. ${ }^{578}$ Despite the potential benefits of centralisation, many drawbacks exist. Potential harm to patients during transport, the loss of medical skills in the regional hospitals, and the separation of patients from family and familiar clinicians are some of the problems that have been raised. ${ }^{6}$ These problems would be surmountable if a centralisation policy for critically ill patients saved lives. This study puts into question the risk:benefit ratio for centralisation, as we detected no benefit on mortality with treatment in high volume units for all admissions with severe sepsis.

\section{Unanswered questions and future research}

Given that variation in outcome is seen across units, future research should potentially focus on other elements of service 
delivery and organisation, beyond volume, used by units with good outcomes. If these elements can be better understood, then other units may be able to implement similar models of care.

\section{Conclusions}

This study found no relation between volume and ultimate acute hospital mortality in admissions with severe sepsis treated in critical care units in the UK.

Contributors: JS, DAH, and KMR contributed to the study design, analysis, and drafting of the manuscript. All the authors approved the final manuscript. JS is the guarantor.

Funding: This research received no specific funding. JS was funded through a bursary provided by the Fonds de Recherche du Québec-Santé.

Competing interests: All authors have completed the Unified Competing Interest form at www.icmje.org/coi_disclosure.pdf (available on request from the corresponding author) and declare: no support from any organisation for the submitted work; no financial relationships with any organisations that might have an interest in the submitted work in the previous three years; no other relationship or activities that could appear to have influenced the submitted work.

Ethical approval: The study had approval by the Ethics Committee at the London School of Hygiene and Tropical Medicine, and ICNARC has approval for the case mix programme database under section 251 of the NHS Act 2006 (approval No PIAG 2-10(f)/2005).

Data sharing: A technical appendix and the statistical code are available from the corresponding author at Jason.shahin@mcgill.ca.

1 Halm EA, Lee C, Chassin MR. Is volume related to outcome in health care? A systematic review and methodologic critique of the literature. Ann Intern Med 2002;137:511-20.

2 Kahn JM. Volume, outcome, and the organization of intensive care. Crit Care 2007;11:129.

3 Christian CK, Gustafson ML, Betensky RA, Daley J, Zinner MJ. The volume-outcome relationship: don't believe everything you see. World J Surg 2005;29:1241-4.

4 Harrison DA, Welch CA, Eddleston JM. The epidemiology of severe sepsis in England, Wales and Northern Ireland, 1996 to 2004: secondary analysis of a high quality clinical database, the ICNARC case mix programme database. Crit Care 2006;10:R42.

5 Nathens AB, Jurkovich GJ, Maier RV, Grossman DC, MacKenzie EJ, Moore M, et al. Relationship between trauma center volume and outcomes. JAMA 2001;285:1164-71.

6 Kahn JM, Branas CC, Schwab CW, Asch DA. Regionalization of medical critical care: what can we learn from the trauma experience? Crit Care Med 2008;36:3085-8.

7 Tilford JM, Simpson PM, Green JW, Lensing S, Fiser DH. Volume-outcome relationships in pediatric intensive care units. Pediatrics 2000;106:289-94.

8 Ramnarayan P, Thiru K, Parslow RC, Harrison DA, Draper ES, Rowan KM. Effect of specialist retrieval teams on outcomes in children admitted to paediatric intensive care units in England and Wales: a retrospective cohort study. Lancet 2010;376:698-704.
9 London Trauma Office. London's trauma system. 2010. www.londontraumaoffice.nhs.uk/ londons-trauma-system/about-the-london-trauma-system/.

10 Angus DC, Black N. Improving care of the critically ill: institutional and health-care system approaches. Lancet 2004;363:1314-20.

11 Peelen L, de Keizer NF, Peek N, Scheffer GJ, van der Voort PH, de Jonge E. The influence of volume and intensive care unit organization on hospital mortality in patients admitted with severe sepsis: a retrospective multicentre cohort study. Crit Care 2007;11:R40.

12 Reinikainen M, Karlsson S, Varpula T, Parviainen I, Ruokonen E, Varpula M, et al. Are small hospitals with small intensive care units able to treat patients with severe sepsis? Intensive Care Med 2010;36:673-9.

13 Parry G, Tucker J, Tarnow-Mordi W. Volume of procedures and outcome of treatment: NHS needs to understand relation more effectively. BMJ 2003;326:280.

14 Knaus WA, Draper EA, Wagner DP, Zimmerman JE. APACHE II: a severity of disease classification system. Crit Care Med 1985:13:818-29.

15 Harrison DA, Parry GJ, Carpenter JR, Short A, Rowan K. A new risk prediction model for critical care: the Intensive Care National Audit \& Research Centre (ICNARC) model. Crit Care Med 2007;35:1091-8.

16 Harrison DA, Brady AR, Rowan K. Case mix, outcome and length of stay for admissions to adult, general critical care units in England, Wales and Northern Ireland: the Intensive Care National Audit \& Research Centre case mix programme database. Crit Care 2004;8:R99-111.

17 The Information Centre. List of databases. 2010. www.icapp.nhs.uk/docdat/DatabaseList. aspx.

18 Royston $\mathrm{P}$, Sauerbrei W. Multivariable model-building: a pragmatic approach to regression analysis based on fractional polynomials for modelling continous variables. John Wiley \& Sons, 2008.

19 Panageas KS, Schrag D, Riedel E, Bach PB, Begg CB. The effect of clustering of outcomes on the association of procedure volume and surgical outcomes. Ann Intern Med 2003;139:658-65.

20 Jones J, Rowan $\mathrm{K}$. Is there a relationship between the volume of work carried out in intensive care and its outcome? Int J Technol Assess Health Care 1995;11:762-9.

21 Goldfrad CK, Rowan K. Does "practice make perfect"? Volume of work and outcome in intensive care [abstract]. J Epidemiol Community Health 2000:54:779

22 Kahn JM, Goss CH, Heagerty PJ, Kramer AA, O'Brien CR, Rubenfeld GD. Hospital volume and the outcomes of mechanical ventilation. N Engl J Med 2006;355:41-50.

23 Kahn JM, Ten Have TR, Iwashyna TJ. The relationship between hospital volume and mortality in mechanical ventilation: an instrumental variable analysis. Health Serv Res 2009;44:862-79.

24 Needham DM, Bronskill SE, Rothwell DM, Sibbald WJ, Pronovost PJ, Laupacis A, et al. Hospital volume and mortality for mechanical ventilation of medical and surgical patients: a population-based analysis using administrative data. Crit Care Med 2006;34:2349-54

25 Castellanos-Ortega A, Suberviola B, García-Astudillo LA, Holanda MS, Ortiz F, Llorca J, et al. Impact of the surviving sepsis campaign protocols on hospital length of stay and mortality in septic shock patients: results of a three-year follow-up quasi-experimental study. Crit Care Med 2010;38:1036-43.

26 May S, Bigelow C. Modeling nonlinear dose-response relationships in epidemiologic studies: statistical approaches and practical challenges. Dose Response 2005;3:474-90.

Accepted: 17 April 2012

\section{Cite this as: BMJ 2012;344:e3394}

This is an open-access article distributed under the terms of the Creative Commons Attribution Non-commercial License, which permits use, distribution, and reproduction in any medium, provided the original work is properly cited, the use is non commercial and is otherwise in compliance with the license. See: $\mathrm{http}: / /$ creativecommons.org/licenses/by$\mathrm{nc} / 2.0 /$ and http://creativecommons.org/licenses/by-nc/2.0/legalcode. 


\section{What is already known on this topic}

A relation between the volume of cases treated and patients' outcomes has been established for several surgical and medical conditions Two small studies have suggested the presence of a volume-outcome relation for critically ill patients with severe sepsis

\section{What this study adds}

No relation was found between volume and acute hospital mortality for patients with severe sepsis admitted to adult general critical care units in the UK

No significant interaction was found between volume and either severity of illness or receipt of mechanical ventilation

\section{Tables}

Table 1| Inclusion criteria for 159483 admissions to 170 adult general critical care units in 2008-09. Values are numbers (percentages) unless stated otherwise

\begin{tabular}{|c|c|c|c|c|}
\hline Criteria & Quarter 1* & Quarter $2^{*}$ & Quarter 3* & Quarter $4^{*}$ \\
\hline All admissions & $(n=22842)$ & $(n=34566)$ & $(n=42728)$ & $(n=59347)$ \\
\hline Median (interquartile range) volume per year & $320(281-374)$ & $432(372-537)$ & $573(465-685)$ & $829(656-1022)$ \\
\hline \multicolumn{5}{|l|}{ No of SIRS criteria met: } \\
\hline 0 & $716(3.1)$ & $829(2.4)$ & $792(1.9)$ & $969(1.6)$ \\
\hline 1 & $2070(9.1)$ & $2853(8.3)$ & $2922(6.8)$ & $4291(7.2)$ \\
\hline 2 & $5672(24.8)$ & $7986(23.1)$ & $9386(22.0)$ & $13108(22.1)$ \\
\hline 3 & $8675(38.0)$ & $13150(38.0)$ & $16690(39.1)$ & $23224(39.1)$ \\
\hline 4 & $5709(25.0)$ & $9748(28.2)$ & $12938(30.3)$ & $17755(29.9)$ \\
\hline Infection & $6203(27.2)$ & 9655 (27.9) & $12497(29.2)$ & $16843(28.4)$ \\
\hline \multicolumn{5}{|l|}{ No of organ dysfunctions: } \\
\hline 0 & $3883(17.0)$ & $4914(14.2)$ & $5904(13.8)$ & $8857(14.9)$ \\
\hline 1 & $7129(31.2)$ & $10457(30.3)$ & $12714(29.8)$ & $18491(31.2)$ \\
\hline 2 & $6423(28.1)$ & $10444(30.2)$ & $12598(29.5)$ & $17560(29.6)$ \\
\hline 3 & $3480(15.2)$ & $5627(16.3)$ & $7402(17.3)$ & $9321(15.7)$ \\
\hline $4-5$ & $1927(8.4)$ & $3124(9.0)$ & $4110(9.6)$ & $5118(8.6)$ \\
\hline Admissions with severe sepsis & $(n=4348 ; 19.0 \%)$ & $(n=7060 ; 20.4 \%)$ & $(n=9390 ; 22.0 \%)$ & $(n=12740 ; 21.5 \%)$ \\
\hline \multicolumn{5}{|l|}{ SIRS criteria: } \\
\hline Temperature & $3156(72.6)$ & $5218(73.9)$ & $6910(73.6)$ & $9588(75.3)$ \\
\hline Heart rate & $4146(95.4)$ & $6733(95.4)$ & $8972(95.5)$ & $12142(95.3)$ \\
\hline Respiratory rate & $4281(98.5)$ & $6991(99.0)$ & 9319 (99.2) & $12602(98.9)$ \\
\hline White blood cell count & $3544(81.5)$ & $5766(81.7)$ & $7650(81.5)$ & $10401(81.6)$ \\
\hline \multicolumn{5}{|l|}{ No of SIRS criteria met: } \\
\hline 3 & $2265(52.1)$ & $3532(50.0)$ & $4709(50.1)$ & $6227(48.9)$ \\
\hline 4 & $2083(47.9)$ & $3528(50.0)$ & $4681(49.9)$ & $6513(51.1)$ \\
\hline \multicolumn{5}{|l|}{ Organ dysfunctions: } \\
\hline Cardiovascular & $3551(81.7)$ & $5810(82.3)$ & $7723(82.2)$ & $10166(79.8)$ \\
\hline Respiratory & $3292(75.7)$ & $5375(76.1)$ & $6966(74.2)$ & 9367 (73.5) \\
\hline Metabolic & $2036(46.8)$ & $3412(48.3)$ & $4781(50.9)$ & $6005(47.1)$ \\
\hline Renal & $1048(24.1)$ & $1857(26.3)$ & 2435 (25.9) & 3405 (26.7) \\
\hline Haematological & $546(12.6)$ & $928(13.1)$ & $1213(12.9)$ & $1753(13.8)$ \\
\hline \multicolumn{5}{|l|}{ No of organ dysfunctions: } \\
\hline 1 & $936(21.5)$ & $1395(19.8)$ & $1892(20.1)$ & $2842(22.3)$ \\
\hline 2 & $1542(35.5)$ & 2527 (35.8) & $3287(35.0)$ & 4491 (35.3) \\
\hline 3 & $1159(26.7)$ & $1871(26.5)$ & $2481(26.4)$ & 3171 (24.9) \\
\hline $4-5$ & $711(16.4)$ & $1267(17.9)$ & $1730(18.4)$ & $2236(17.6)$ \\
\hline
\end{tabular}

SIRS=systemic inflammatory response syndrome. 
Table 1 (continued)

Criteria

*Volume of admissions with severe sepsis grouped by quarters of critical care units. 
Table 2| Volume characteristics of 33538 admissions with severe sepsis between 2008 and 2009 by critical care unit volume and admission volume

\begin{tabular}{|c|c|c|c|c|}
\hline Characteristic & Quarter 1 & Quarter 2 & Quarter 3 & Quarter 4 \\
\hline \multicolumn{5}{|l|}{ Distribution by critical care unit } \\
\hline Total No of admissions & 4348 & 7060 & 9390 & 12740 \\
\hline Total No of units & 43 & 42 & 43 & 42 \\
\hline Median (IQR) volume of admissions with severe sepsis per unit per year & $70(59-75)$ & $98(95-103)$ & $130(121-138)$ & $190(168-206)$ \\
\hline No (\%) hospital type: university or university affiliated & $10(23)$ & $13(31)$ & $19(44)$ & $28(67)$ \\
\hline Median (IQR) No of beds in unit & $6(5-7)$ & $8(7-9)$ & $10(8-12)$ & $14(12-17)$ \\
\hline \multicolumn{5}{|l|}{ Distribution by admission } \\
\hline Total No of admissions & 8544 & 8337 & 8435 & 8222 \\
\hline Total No of units & 70 & 41 & 34 & 25 \\
\hline Median (IQR) volume of admissions with severe sepsis per unit per year & $84(68-95)$ & $119(105-126)$ & $150(141-166)$ & $195(191-212)$ \\
\hline No (\%) hospital type: university or university affiliated & $21(30)$ & $9(22)$ & $24(71)$ & $16(64)$ \\
\hline Median (IQR) No of beds in unit & $7(6-8)$ & $8(7-10)$ & $12(10-15)$ & $16(13-18)$ \\
\hline
\end{tabular}




\begin{tabular}{|c|c|c|c|c|}
\hline Characteristic & Quarter 1* (n=3977) & Quarter 2* $(n=6490)$ & Quarter $3^{*}(n=8619)$ & Quarter $4^{*}(n=11641)$ \\
\hline \multicolumn{5}{|l|}{ Demographics } \\
\hline Mean (SD) age & $64.8(16.2)$ & $64.0(16.4)$ & $63.7(16.7)$ & $63.1(16.7)$ \\
\hline Male sex & $2099(52.8)$ & $3454(53.2)$ & $4569(53.0)$ & $6371(54.7)$ \\
\hline White ethnicity & $3734(93.9)$ & $5861(90.3)$ & $7781 / 8493(91.6)$ & $10412(89.4)$ \\
\hline \multicolumn{5}{|l|}{ Acute severity of illness } \\
\hline Median (IQR) APACHE II score† & $19(14-23)(n=3867)$ & $19(15-24)(n=6302)$ & $19(15-24)(n=8384)$ & $19(15-24)(n=11374)]$ \\
\hline Median (IQR) ICNARC physiology score & $22(17-29)$ & $23(17-30)$ & $22(16-29)$ & $22(16-28)$ \\
\hline \multicolumn{5}{|l|}{ Medical history } \\
\hline Severe comorbidities $\ddagger:$ & $(n=3950)$ & $(n=6450)$ & $(n=8571)$ & $(n=11602)$ \\
\hline Any previous illness & $600(15.2)$ & $1060(16.4)$ & $1512(17.6)$ & $2333(20.1)$ \\
\hline Severe cardiovascular disease & $87(2.2)$ & $76(1.2)$ & $141(1.6)$ & $164(1.4)$ \\
\hline Severe respiratory disease & $148(3.7)$ & $245(3.8)$ & $303(3.5)$ & $373(3.2)$ \\
\hline Renal disease & $21(0.5)$ & $81(1.3)$ & $131(1.5)$ & $291(2.5)$ \\
\hline Chronic liver disease & $50(1.3)$ & $103(1.6)$ & $179(2.1)$ & $277(2.4)$ \\
\hline Haematological malignancy & $136(3.4)$ & $216(3.3)$ & $281(3.3)$ & $491(4.2)$ \\
\hline Metastatic disease & $76(1.9)$ & $111(1.7)$ & $164(1.9)$ & $251(2.2)$ \\
\hline Immunological dysfunction & $230(5.8)$ & $422(6.5)$ & $595(6.9)$ & $984(8.5)$ \\
\hline Activities of daily living§: & $(n=3948)$ & $(\mathrm{n}=6447)$ & $(\mathrm{n}=8480)$ & $(n=11602)$ \\
\hline No assistance & $2997(75.9)$ & $4615(71.6)$ & $6055(71.4)$ & $8319(71.7)$ \\
\hline Partial assistance & $897(22.7)$ & $1763(27.4)$ & $2309(27.2)$ & $3158(27.2)$ \\
\hline Total assistance & $54(1.4)$ & $69(1.1)$ & $116(1.4)$ & $125(1.1)$ \\
\hline Cardiac arrest $\emptyset$ & $137(3.4)$ & $279(4.3)$ & $300(3.5)$ & $337(2.9)$ \\
\hline \multicolumn{5}{|l|}{ Admission variables } \\
\hline Location before admission ${ }^{\star *}$ : & & $(\mathrm{n}=6489)$ & & \\
\hline Emergency/urgent surgery & $810(20.4)$ & $1580(24.4)$ & $2170(23.2)$ & $2666(22.9)$ \\
\hline Elective/scheduled surgery & $172(4.3)$ & $231(3.6)$ & $396(4.6)$ & $514(4.4)$ \\
\hline Hospital ward & $1968(49.5)$ & $3119(48.1)$ & $4146(48.1)$ & $5825(50.0)$ \\
\hline High dependency unit & $348(8.8)$ & $515(7.9)$ & $520(6.0)$ & $697(6.0)$ \\
\hline Emergency department & $676(17.0)$ & $1042(16.1)$ & $1385(16.1)$ & $1933(16.6)$ \\
\hline Mechanical ventilation & $2345 / 3975(59.0)$ & $4035 / 6488(62.2)$ & $5242 / 8618(60.8)$ & $6562 / 11637(56.4)$ \\
\hline \multicolumn{5}{|l|}{ Length of stay: } \\
\hline Median (IQR) days of unit stay & $4.6(1.9-10.7)$ & $4.1(1.8-9.4)$ & $4.0(1.8-9.1)$ & $4.1(1.9-9.3)(n=11639)$ \\
\hline Median (IQR) days of hospital stay & $17(8-33)$ & $17(8-34)$ & $17(8-35)$ & $19(9-37)(n=11639)$ \\
\hline \multicolumn{5}{|l|}{ Mortality: } \\
\hline Unit mortality & $1242(31.2)$ & $1973(30.4)$ & $2527(29.3)$ & $3326(28.6)$ \\
\hline Ultimate acute hospital mortality & $1682 / 3940(42.7)$ & $2722 / 6396(42.6)$ & $3456 / 8563(40.4)$ & $4504 / 11561(39.0)$ \\
\hline Hospital discharge location††: & $(n=2063)$ & $(\mathrm{n}=3302)$ & $(n=4662)$ & $(n=6590)$ \\
\hline $\begin{array}{l}\text { Home, residential place of } \\
\text { work/education, or non-health related } \\
\text { institution }\end{array}$ & $1849(89.6)$ & $2864(86.7)$ & $4057(87.0)$ & $5854(88.8)$ \\
\hline Nursing home or equivalent & $62(3.0)$ & $108(3.3)$ & $168(3.6)$ & $196(3.0)$ \\
\hline Short term rehabilitation & $105(5.1)$ & $231(7.0)$ & $333(7.1)$ & $323(4.9)$ \\
\hline Long term rehabilitation & $24(1.2)$ & $49(1.5)$ & $64(1.4)$ & $131(2.0)$ \\
\hline Other health related institution & $15(0.7)$ & $37(1.1)$ & $29(0.6)$ & $61(0.9)$ \\
\hline Hospice or equivalent & $8(0.4)$ & $13(0.4)$ & $11(0.2)$ & $25(0.4)$ \\
\hline
\end{tabular}

APACHE=acute physiological and chronic health evaluation; ICNARC=Intensive Care National Audit \& Research Centre; IQR=interquartile range. *Volume of admissions with severe sepsis grouped by quarters of critical care units.

$\dagger 1327$ admissions not eligible for calculation of APACHE II. ${ }^{28}$ 


\section{Table 3 (continued)}

\section{Characteristic}

$$
\text { Quarter } 1^{*}(n=3977)
$$

¥Severe cardiovascular disease was defined as New York Heart Association class IV angina; severe respiratory disease as shortness of breath with light activity due to a pulmonary disorder or chronic home ventilatory support; renal disease as receipt of chronic peritoneal dialysis or haemodialysis; chronic liver disease as portal hypertension or hepatic encephalopathy or biopsy proven cirrhosis; haematological malignancy as any evidence of acute or chronic myelogenous leukaemia, acute or chronic lymphocytic leukaemia, or lymphoma; metastatic disease as evidence of distant metastases to areas other than regional lymph nodes; and immunological dysfunction as congenital immunohumoral or cellular immune deficiency states or receipt of chemotherapy or prednisone. Excluding admissions with no evidence to assess past medical history.

§Functional status was assessed by how much assistance was needed to carry out activities of daily living and defined as no assistance, partial assistance, or total assistance. Excluding admissions with no evidence to assess past medical history.

TDefined as receipt of cardiopulmonary resuscitation in 24 hours before admission.

**Thirteen admissions direct from home not reported.

††Hospital survivors only. Excluding admissions transferred to another acute hospital. 
Table 4 | Relation between volume of admissions with severe sepsis per year and mechanical ventilation status by different volume groupings

\begin{tabular}{lc} 
Grouping and quarter & Strata specific odds ratio $(95 \%$ Cl) \\
\begin{tabular}{l} 
Volume grouped by admissions \\
\hline No mechanical ventilationt:
\end{tabular} \\
\hline 1 & 1.0 \\
\hline 2 & $0.91(0.77$ to 1.06$)$ \\
\hline 3 & $0.94(0.79$ to 1.11$)$ \\
\hline 4 & $0.93(0.78$ to 1.11$)$ \\
\hline Mechanically ventilation: & 1.0 \\
\hline 1 & $1.0(0.87$ to 1.15$)$ \\
\hline 2 & $0.92(0.79$ to 1.07$)$ \\
\hline 3 & $0.92(0.79$ to 1.08$)$ \\
\hline 4 & \\
\hline Volume grouped by unitst & \\
\hline No mechanical ventilation: & 1.0 \\
\hline 1 & $1.17(0.96$ to 1.42$)$ \\
\hline 2 & $0.96(0.80$ to 1.17$)$ \\
\hline 3 & $1.04(0.86$ to 1.25$)$ \\
\hline 4 & \\
\hline Mechanically ventilation: & 1.0 \\
\hline 1 & $0.84(0.71$ to 0.99$)$ \\
\hline 2 & $0.86(0.73$ to 1.02$)$ \\
\hline 3 & $0.84(0.71$ to 0.99$)$ \\
\hline 4 & \\
\hline
\end{tabular}

${ }^{*}$ Calculated using likelihood ratio test comparing model with no interaction term to one with interaction term.

†Model adjusted for volume age, sex, ICNARC physiology score, comorbidities, activities of daily living, cardiac arrest, location before admission, and hospital type; an interaction term was placed between unit admission volume and mechanical ventilation. 


\section{Figures}

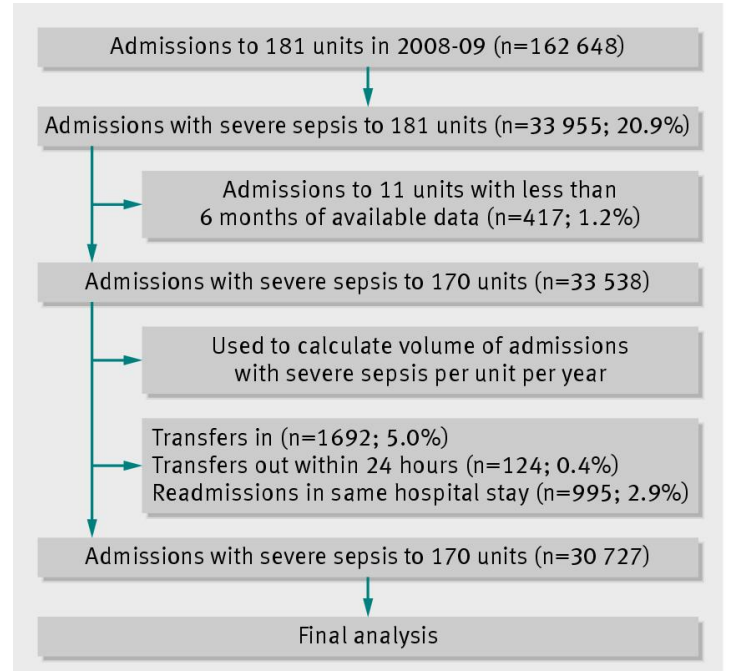

Fig 1 Flow chart of admissions and critical care units

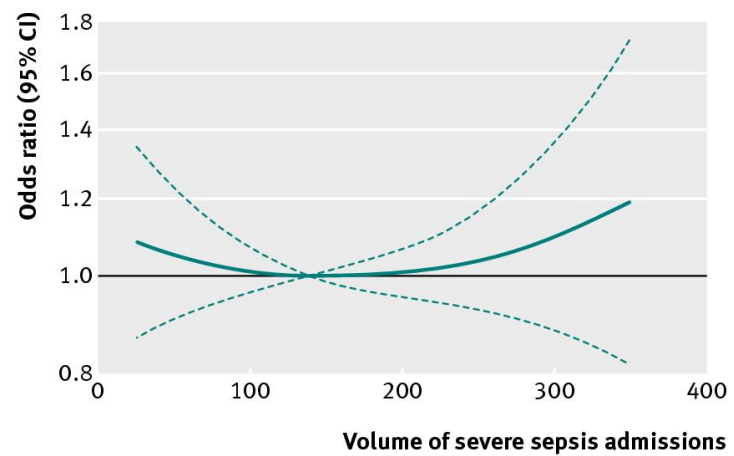

Fig 2 Odds ratio $(95 \% \mathrm{Cl})$ for effect of volume on acute hospital mortality. Volume modelled using fractional polynomials (degree 2) relative to mean volume of 138 admissions per year. Model adjusted for age (fraction polynomials degree 2), sex, ICNARC physiology score (fractional polynomials degree 2), mechanical ventilation, comorbidities, activities of daily living, cardiac arrest, location before admission, and hospital type 




Fig 3 Odds ratio $(95 \% \mathrm{Cl})$ for interaction between effect of volume and mechanical ventilation on acute hospital mortality. Volume modelled using fractional polynomials (degree 2) relative to mean volume of 138 admissions per year. Model adjusted for age (fraction polynomials degree 2), sex, ICNARC physiology score (fractional polynomials degree 2), mechanical ventilation, comorbidities, activities of daily living, cardiac arrest, location before admission, and hospital type
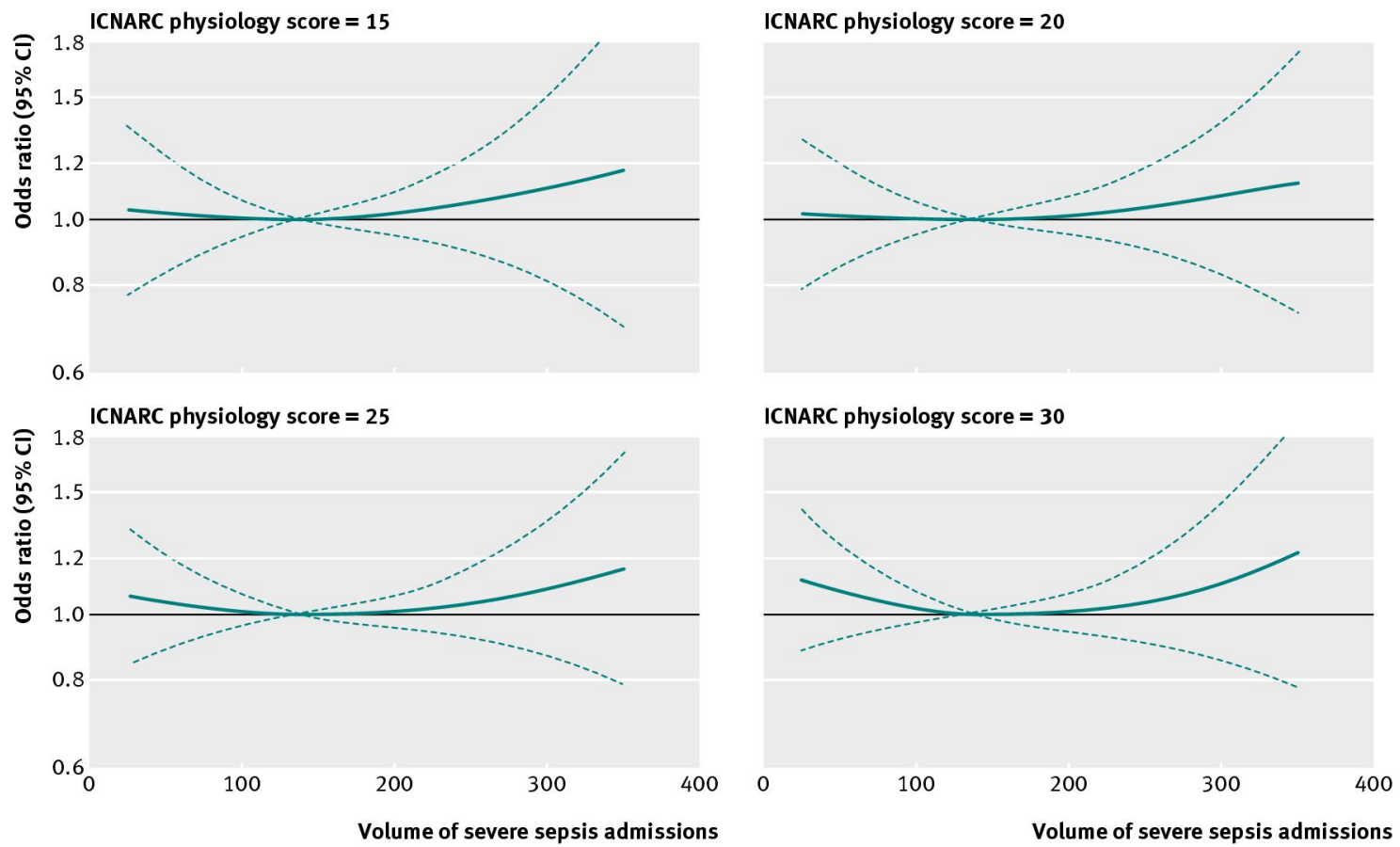

Fig 4 Odds ratio $(95 \% \mathrm{Cl})$ for interaction between effect of volume and Intensive Care National Audit \& Research Centre (ICNARC) physiology score on acute hospital mortality. Volume modelled using fractional polynomials (degree 2 ) relative to mean volume of 138 admissions per year. Model adjusted for age (fraction polynomials degree 2), sex, ICNARC physiology score (fractional polynomials degree 2), mechanical ventilation, comorbidities, activities of daily living, cardiac arrest, location before admission, and hospital type 